\title{
Directional connectivity in the EEG is able to discriminate wakefulness from NREM sleep
}

\author{
G Lioi $^{1 *}$, S L Bell ${ }^{1}$, D C Smith ${ }^{1,2}$ and DM Simpson ${ }^{1}$ \\ ${ }^{1}$ Institute for Sound and Vibration Research, University of Southampton, Southampton, UK \\ ${ }^{2}$ Department of Anesthesia, University Hospital Southampton NHS Foundation Trust, Southampton, UK
}

\begin{abstract}
A reliable measure of consciousness is of great interest for various clinical applications including sleep studies and the assessment of depth of anaesthesia. A number of measures of consciousness based on the EEG have been proposed in the literature and tested in studies of dreamless sleep, general anaesthesia and disorders of consciousness. However, reliability has remained a persistent challenge. Despite considerable theoretical and experimental effort, the neural mechanisms underlying consciousness remain unclear, but connectivity between brain regions is thought to be disrupted, impairing information flow. The objective of the current work was to assess directional connectivity between brain regions using Directed Coherence and propose and assess an index that robustly reflects changes associated with non-REM sleep. We tested the performance on polysomnographic recordings from ten healthy subjects and compared Directed Coherence (and derived features) with more established measures calculated from EEG spectra. We compared the performance of the different indexes to discriminate the level of consciousness at group and individual level. At a group level all EEG measures could significantly discriminate NREM sleep from waking, but there was considerable individual variation. Across all individuals, normalized power, the strength of long-range connections and the direction of functional links strongly correlate with NREM sleep stages over the experimental timeline. At an individual level, of the EEG measures considered, the direction of functional links constitutes the most reliable index of the level of consciousness, highly correlating with the individual experimental time-line of sleep in all subjects. Directed Coherence thus provides a promising new means of assessing level of consciousness, firmly based on current physiological understanding of consciousness.
\end{abstract}

Keywords: EEG, Functional Connectivity, Directed Coherence, Sleep, Consciousness.

\footnotetext{
${ }^{*}$ Corresponding author at: Institute for Sound and Vibration Research, University of Southampton, Southampton SO17 1BJ, UK. Tel: +44 7405258497. E-mail address: g14g13@ soton.ac.uk; lioi.giulia@gmail.com (G. Lioi).
} 


\section{Introduction}

Neural correlates of consciousness have attracted considerable interest in recent years (De Graaf, Hsieh and Sack, 2012). This has motivated a series of studies that contrast brain activity in wakefulness, where consciousness is typically present, with conditions where it is diminished or suppressed, such as in dreamless sleep (Massimini et al., 2005; Spoormaker et al., 2010; Siclari et al., 2014) or anaesthesia (Ferrarelli et al., 2010; Gómez et al., 2013). In parallel, a series of theoretical advances have predicted that consciousness is critically related to functional connectivity that enables widespread information sharing among distant brain areas (Tononi, 2008). A series of putative markers of consciousness have been proposed in the literature that include event related potentials evoked by auditory or visual stimuli (Sergent, Baillet and Dehaene, 2005; Sitt et al., 2014), spectral patterns (Mhuircheartaigh et al., 2013) gamma synchrony and measures of information sharing across distant brain areas (Tononi and Massimini, 2008). A recent critical review (Koch et al., 2016) has highlighted the importance of EEG as a fundamental clinical tool to discriminate conscious from unconscious subjects, however, it has suggested that only few of these measures are promising neurophysiology correlates of consciousness. The low-frequency high amplitude EEG (usually referred to as slow-waves) that characterizes the loss of consciousness in physiological, drug-induced or pathological conditions, as opposite to high-frequency "Activated-EEG" in wakefulness, remains one of the oldest and most reliable markers of awareness (Koch et al., 2016). Other promising approaches appear to be measures of brain connectivity. These are thought to indicate the ability of the brain to integrate information (Tononi, 2008).

Consciousness naturally fades during deep non-rapid eye movement (NREM) sleep, in particular in the early night (Tononi and Massimini, 2008), when reports after awakening refer to little or absent conscious experience (Stickgold et al., 2001); thus the onset of NREM sleep may represent an opportunity to relate changes in brain activity to changes in consciousness. During REM sleep, in contrast, reports of dream-like experiences are common. A nap generally includes only NREM sleep $(\mathrm{N} 1, \mathrm{~N} 2$ and $\mathrm{N} 3$ ) with sleep $\mathrm{N} 2$ representing the largest proportion and the time spent in N3 increasing with the nap-length for naps longer than 10 minutes (Brooks and Lack, 2006). Naps , as also used by Massimini and Tononi (Massimini et al., 2005), therefore represent a convenient condition to investigate loss of consciousness at the onset of NREM sleep, with the advantage of only requiring a simple experimental setup that does not entail the practical challenges of overnight sleep recordings.

Changes in cortical connectivity associated with sleep have been widely investigated in functional magnetic resonance (fMRI) studies, but their relationship with consciousness remains unclear (Klimova, 2014). Results point to a general impairment of functional connectivity in the thalamocortical system (Spoormaker et al., 2010; Spoormaker, Gleiser and Czisch, 2012); in particular, long-range connectivity was shown to be affected by sleep (Tagliazucchi et al., 2013) and connectivity networks in NREM sleep show increased local clustering when compared to wakefulness (Boly et al., 2012). Recent studies investigating early NREM sleep with combined transcranial magnetic stimulation (TMS) and EEG approaches (Massimini et al., 2005) show a break-down of large-scale connectivity in the sleeping brain.

EEG is often preferred in studies of brain connectivity from a practical point of view (Sitt et al., 2014), as it can be applied relatively easily at the bed-side and at low cost, and EEG-based systems can be used in routine clinical work in the home or ward, as well as in intensive care units or operating theatres where assessment of level of consciousness (LOC) may be carried out. The strength of frequency-dependent relationships between EEG channels in sleep have mainly been investigated in previous studies with conventional Coherence $(\mathrm{COH})$ estimates (Achermann and Borbély, 1998; Corsi-Cabrera et al., 2003) and Synchronicity (Ferri et al., 2005, 2007). COH and Synchronicity are symmetrical measures unable to convey directional information, therefore losing some functional 
significance. A more advanced measure of functional connectivity, the Directed Coherence DC (Baccalá et al. 1998) has been proposed to overcome this limitation, by using a model-based approach involving causal linear filters quantifying the interactions between channels. The DC estimator is consistent with the framework of Granger Causality and provides information about the strength, direction and spectral content of linear dependencies. It therefore has potential to give the additional information about the direction of functional links in the brain compared to standard $\mathrm{COH}$. It has been demonstrated that for Gaussian and quasi-Gaussian distributions (Hlaváčková-Schindler, 2011), GC is equivalent to transfer entropy, but has the advantage of being simpler to understand and interpret and easier to apply, providing a straightforward decomposition in frequency (Barnett, Barrett and Seth, 2009). This property is of particular relevance for EEG applications, where specific brain rhythms are dominant in behavioural states such as sleep stages, or when performing cognitive tasks (Klimesch, 1999).

The aim of the current study is therefore to describe changes in strength and direction of functional connectivity associated with NREM sleep using Directed Coherence and to propose and test indexes of brain connectivity based on DC that could distinguish between states of consciousness. Performance of the proposed approaches will be compared to more established spectral measures and assessed in discriminating between NREM sleep and wakefulness. Since the overarching aim of any proposed measure is to assess the LOC in individual subjects, performance is assessed against each subject's own time-line through the sleep stages. The focus on individual variability is an important and distinctive feature of this work, since the majority of previous studies investigating correlates of consciousness (where consciousness is diminished or reduced) have focussed on the analysis of the average values across the cohort. This work is also original in investigating directional connectivity (using Directed Coherence) on EEG, where previously the strength of connection, rather than the direction of information flow, was the focus. Our interest in individual variability is motivated by a potential clinical application: we hope to assess depth of anaesthesia in future work and so we are looking for an index that is computationally convenient and suitable for online monitoring of individuals and can show changes in individual subjects, not just statistical differences between groups. In this sense the spontaneous EEG is also preferable to fMRI and TMS approaches as it represents a low cost and easily implementable method with good temporal resolution.

\section{Methods}

\subsection{Subjects, Protocol and Preprocessing}

Sixteen healthy subjects participated in the sleep study. However only ten subjects (three females and seven males, aged between 22 and 30 years) underwent N1, N2 and N3 stages of NREM sleep and so were included in the analysis. The experiment was approved by the local ethics committee and following informed consent, and conformed to requirements of the Declaration of Helsinki. In order to exploit the circadian sleep drive, the experiment was performed in the afternoon after lunch. The subjects were asked to refrain from drinking coffee or tea on the day of the experiment. The subjects, lying with eyes closed on a reclining chair, were invited to sleep. After they spontaneously woke up again, they were asked to rest with eyes closed (REST W) and then to perform mental arithmetic with eyes closed (ACTIVE W). EEG was collected using a 32 channel system with active electrodes (Biosemi BV, Amsterdam) placed according to the international 10-20 system. Additional electrodes were used to record the electrooculogram (EOG) and the chin electromyogram (EMG). Sleep stages were scored by visual inspection of contiguous epochs of $30 \mathrm{~s}$ according to the standard criteria (Iber et al. 2007). Data were then downsampled to $250 \mathrm{~Hz}$ and digitally referenced with respect to the average of T7 and T8 channels (linked mastoid), as recommended for functional connectivity estimation (Kaminski and Blinowska, 2014). The EEG time series were band pass filtered (1-45 Hz) 
and additionally notch filtered at the mains frequency using zero phase filters. Only continuous and artifact-free epochs were selected and included in the following analysis.

\subsection{Multivariate connectivity estimation}

The DC is obtained from multivariate autoregressive (MVAR) model parameters. A MVAR process describes each multi-channel EEG time series $\boldsymbol{x}$ as a sum of $p$ previous samples from the set of Msignals (here the EEG channels), weighted by model coefficients, plus a noise component, as given in the following equation (1):

$$
\boldsymbol{x}(n)=\sum_{l=1}^{p} \boldsymbol{A}(l) \boldsymbol{x}(n-l)+\boldsymbol{\varepsilon}(n), n=1, \ldots \boldsymbol{N}
$$

where $\boldsymbol{x}(n)$ is the $\mathrm{M}$ dimensional vector of the EEG channels time-series at time lag $n, N$ is the number of samples in the signals, $\boldsymbol{A}(l)$ is the $M x M$ coefficient matrix (weights) describing the linear interactions between channels at lag $l, p$ is the model order, and $\varepsilon(n)$ is the vector of white innovations, with the non-singular residual covariance matrix:

$$
\Sigma_{\varepsilon}=\left\{\sigma_{i j}^{2}\right\}
$$

$\sigma_{i j}^{2}$ is the cross-covariance between innovations signals $\boldsymbol{\varepsilon}(n)$ for channels $i$ and $j$.

The weights relate the present sample of one signal to the past of another (and itself) and capture the directed influence between signals that can be interpreted in the sense of the Granger Causality (Granger, 1969). It should further be pointed out that equation (1) explicitly excludes instantaneous connections (with zero time-lag). By transformation into the frequency domain, the MVAR process is modelled as a filter with transfer matrix $\boldsymbol{H}(f)$ and white noise $\boldsymbol{E}(f)$ as an input:

$$
\boldsymbol{X}(f)=\boldsymbol{A}(f) \boldsymbol{X}(f)+\boldsymbol{E}(f)=\boldsymbol{H}(f) \boldsymbol{E}(f)
$$

In equation (3), $\boldsymbol{A}(f)$ is the Fourier Transform of the matrix of parameters and $\boldsymbol{H}(f)=[\boldsymbol{I}-\boldsymbol{A}(f)]^{-1}$ (where $\boldsymbol{I}$ is the identity matrix), which conveys information about the linear dependencies between signals and their spectral features. The DC from signal $j$ to signal $i$ of the M-variate dataset is defined as follows (Baccalá et al. 1998)

$$
D C_{i, j}(f)=\frac{\sigma_{j j} H_{i, j}(f)}{\sqrt{\sum_{m=1}^{M} \sigma_{m m}^{2}\left|H_{i, m}(f)\right|^{2}}}
$$

and, because of the normalization, it quantifies the linear coupling from $x_{j}$ to $x_{i}$ as compared to all the other contributions the signal $x_{i}$ receives from other structures of the $\mathrm{M}$-variate dataset. In particular it has been shown (Faes et al., 2013) that the squared modulus of $D C_{i, j}(f)$ measures the normalized portion of the autospectrum of $x_{i}$ at frequency $f$ due to the signal $x_{j}$ (or transferred from $x_{j}$ via the transfer function $H_{i, j}(f)$ to $x_{i}$ ). In other words $\left|D C_{i, j}(f)\right|^{2}$ is a measure of the portion of the autospectrum of $x_{i}$ at frequency $f$ due to the signal $x_{j}$. Due to its relatively straightforward interpretation in term of spectral content, in this work we will use this squared modulus of DC to quantify functional connectivity. This differs from the related formulation of the Directed Transfer Function (DTF, Kaminiski and Blinowska 1991); as DC includes the variance of the residuals $\sigma_{*}$, it brings the advantage of robustness against different signal scaling (Baccalà and Sameshima, 2007). When all residuals variances are equivalent, the DC reduces to the DTF. One may view this as DTF reflecting the existence of (directional) connections, while DC also quantifies how these connections are used and it is interpretable in terms of signal power content. 
When estimating an MVAR model of order $p$ from a dataset, it is important that segments of EEG data of adequate length are collected to ensure that the number of samples is sufficient to accurately fit the model. Given a M-variate dataset, a minimum of $M^{2} p$ data points is required for the model fitting, since there are $M^{2} p$ parameters to estimate; however in practice a much higher number is recommended (typically 10 times the minimum number) for an accurate estimate (Schlogl and Supp, 2006). In order to follow this recommendation and to reduce computational cost, which is always of concern in multivariate connectivity estimation, a reduced number of electrodes was considered for connectivity analysis, as did Toppi et al. (2012) and Marinazzo et al. (2014). A subset of $M=12$ electrodes that are fairly evenly distributed across the scalp (Fp1, Fp2, F3, Fz, F4, C3, Cz, C4, P3, P4, O1, O2) was selected and connectivity was estimated for epochs of 60 s (i.e. 15000 samples in each channel) that were not interrupted by artefacts or high-level noise (according to visual inspection). Therefore only segments with two consecutive $30 \mathrm{~s}$ epochs of the same sleep stage were included in the study while isolated epochs were excluded from the following analysis.

When making inferences about EEG connectivity, only statistically significant estimates should be considered. In this study the significance of DC links was assessed using surrogate data based on a phase shuffling of the EEG signals, with 1000 repetitions that generated a set of surrogate data in which any temporal correlation between channels was removed, but autocorrelation (and thus the spectrum) of each signal was maintained. DC was then estimated from the surrogate dataset in order to obtain an empirical null distribution for each pair of signals at all frequencies. The significance of causal links was assessed comparing the estimated connectivity with the null distribution, setting the significance level at $\mathrm{p}<0.01$. Correction for multiple comparisons was performed using the false discovery rate (FDR) approximation for dependent measurements (Benjamini and Yekutieli, 2001). Only links that were thus found to be statistically significant were included in the subsequent analysis and in the calculation of EEG indexes of connectivity.

A widespread practice in functional connectivity analysis is to threshold connectivity matrices to remove weak or spurious connections and retaining only a small percentage of the strongest connections (Sporns, 2013). In this study the connectivity matrices were thresholded to retain either $10 \%$ or $30 \%$ of the strongest connections (as in Chennu et al., 2014) and then averaged in the four physiologically relevant frequency bands delta $(\delta)[1-4] \mathrm{Hz}$, theta $(\theta)$ [4-7] $\mathrm{Hz}$, alpha $(\alpha)[8-13] \mathrm{Hz}$ and beta $(\beta)[13-25] \mathrm{Hz}$.

In order to specifically investigate changes in long-range connectivity, the DC links were subdivided with respect to the 3D Euclidian interelectrode distances, computed using default channel coordinates. Distance thresholds were set for differentiating between three groups of channel pairs in roughly the same proportions with respect to the total number of possible links: $35 \%$ of short-range links (interchannel distances below $10 \mathrm{~cm}$ ), 32\% of medium range links (between 10 and $14 \mathrm{~cm}$ ) and $33 \%$ of long-range links (above $14 \mathrm{~cm}$ ).

\subsection{Computation of EEG indexes}

For each $60 \mathrm{~s}$ epoch of different sleep stages and for each subject, a series of EEG measures were then extracted. We organized indexes into two classes: spectral measures (as commonly used in many previous studies) and connectivity based measures. The power spectral density (PSD) for each epoch and electrode was estimated using the Welch method (Hanning window $7.5 \mathrm{~s}$ long, 50\% overlap); the power in each frequency band was calculated as the integral of the PSD within each frequency band. The spectral analysis was focused on $\delta, \theta$ and $\alpha$ bands since previous studies on neural correlates of consciousness had reported major changes in these bands (Chennu et al., 2014; Koch et al., 2016). To allow for differences in power between EEG channels, we estimated the normalized power in these three frequency bands by the total power $(1-45 \mathrm{~Hz})$ in each time epoch and for each electrode. The 
normalized power is thought to be a more reliable estimator because it encompasses the individual variances in the absolute EEG power caused, for instance, by variations in electrodes impedances (Sitt et al., 2014).

We assessed connectivity through indexes quantifying the strength of the connectivity networks and indexes estimating the direction of information flow. The rationale for the former is to be found in the large number of studies showing that long-range connectivity is significantly affected by the LOC. The rationale for the latter comes from published results showing a prevalence of frontal EEG activation in sleep as opposed to a strong posterior activation in wakefulness (Brown, Lydic and Shiff, 2010), with indications that the direction of long-range connectivity may represent a prominent feature of sleep as compared to wakefulness (De Gennaro et al., 2004). To this end we assessed the number of significant connections from centro-posterior $(\mathrm{O} 1, \mathrm{O} 2, \mathrm{P} 3, \mathrm{P} 4, \mathrm{C} 3, \mathrm{C} 4, \mathrm{Cz})$ to anterior (Fp1, Fp2, F3, F4, Fz) electrodes and vice versa. We thus defined an index that quantifies the dominant direction of information flow on the front-posterior axis $\left(\operatorname{Dir}_{P-\rightarrow \mathrm{A}}\right)$ as the normalized differences of the number of links in the two opposite directions over the rostro-caudal axis:

$$
\operatorname{Dir}_{P \rightarrow>A}=\frac{\sum_{i} \sum_{j} n u m\left(D C_{i j P->A}\right)-\sum_{i} \sum_{j} n u m\left(D C_{i j A->P}\right)}{\sum_{i} \sum_{j} n u m\left(D C_{i j P->A}\right)+\sum_{i} \sum_{j} n u m\left(D C_{i j A->P}\right)}
$$

where the sums are taken over all pairwise connections between the posterior and anterior channels $(\mathrm{P}$ $\rightarrow \mathrm{A})$ and anterior to posterior channels $(\mathrm{A} \rightarrow \mathrm{P})$, respectively and $n u m()=$.1 when that connection is significant, and zero otherwise.

In the following, we will first present results at a group (cohort) level, showing differences in connectivity based measures between the different sleep stages and frequency bands, with associated statistical analysis (Friedman tests). The average topographic distribution of spectral parameters and connectivity measures is then assessed and the ability of these and derived indexes to distinguish between sleep stages at a group-level is tested. We then also present results at an individual subject level, and the most promising measures are correlated with the manual scoring of sleep stages (hypnogram) in each individual, by Spearman correlation using the indexes from consecutive 1minute segments. The presentation of results from individual subjects, in addition to the statistical analysis across the cohort, provides insight into the potential of the method in monitoring individual patients.

\section{Results}

\subsection{Group analysis}

According to standard criteria (Iber et al. 2007), hypnograms were generated for each subject, using consecutive one-minute artefact free signal segments. The hypnograms represent the sleep stages visually identified from off-line scoring, and their assessment was carried out blinded to the connectivity analysis that follows. The following percentages of time spent in the sleep and wakefulness stages were obtained across subjects: sleep N3 21 $\pm 9 \%$, sleep N2 $27 \pm 4 \%$, sleep N1 $16 \pm 7 \%$, REST W $19 \pm 6 \%$, and ACTIVE W $16 \pm 4 \%$. Since volunteers were allowed to spontaneously wake up from the post-prandial sleep, sleep duration was highly variable across subjects $(16.2 \pm 5.9$ minutes of sleep were analysed per subject).

We calculated the average strength of DC links in the different experimental stages and then averaged this across subjects. Figure 1 shows DC average strength (across subjects and electrodes) as a function of the distance threshold and the sleep stages. The strength of connectivity links exhibit different 
trends across sleep stages depending on the distance range considered. Long-range connections are generally disrupted in NREM sleep where connectivity networks gain a more localized character (there is a prevalence of short-range links in sleep $\mathrm{N} 2$ and $\mathrm{N} 3$, in particular in the $\delta$ band) and longrange connectivity in the $\alpha$ band showed the best performance in distinguishing sleep from wakefulness.. This is in accordance with previous works (Chennu et al., 2014; Lioi, Bell and Simpson, 2016). In order to reduce the number of parameters investigated and for the sake of the clarity, we therefore only included indexes relative to the $\alpha$ band as markers of connectivity in the later results.
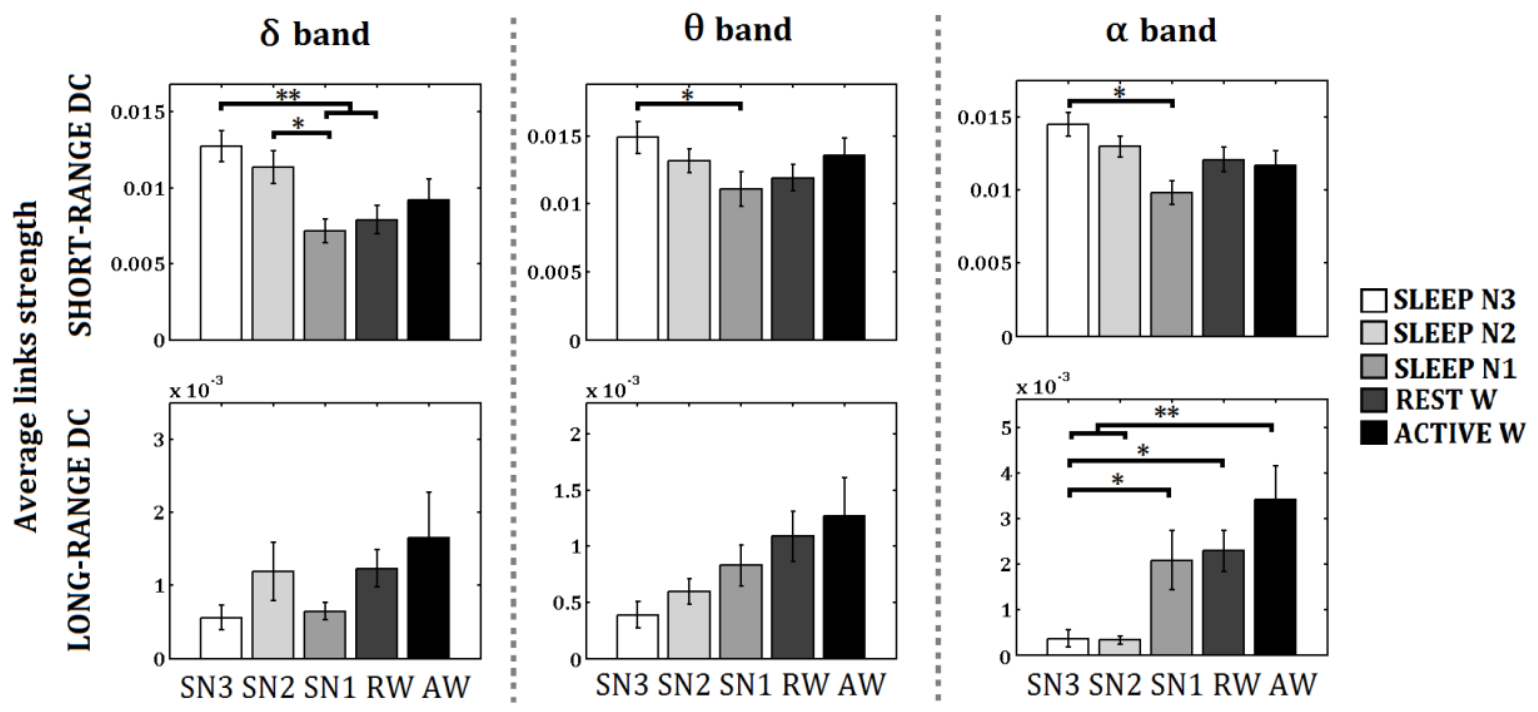

Figure 1. Short and long-range connectivity (as measured by Directed Coherence) in $\delta, \theta$ and $\alpha$ bands. Top bar row: short-range connections. Bottom row: long-range connections. Bars indicate average strength of $10 \%$ strongest DC (magnitude squared) links across subjects $(\mathrm{N}=10)$. The error bars represent the within group standard error. The asterisks specify that the two means designated by the brackets significantly differ (Friedman test with post-hoc analysis, $* \mathbf{p}<0.05, * * \mathbf{p}<0.01)$. Short-range connectivity is dominant in NREM sleep $(\mathrm{N} 2+\mathrm{N} 3)$ while the strength of long-range links is reduced as compared to wakefulness, in particular in the $\alpha$ band.

Figure 2 shows the group topographic characteristics of a number of features derived from the EEG (band power, strength of long range functional connections and the direction of functional connections) as a function of sleep stages, along with mean and standard error plots for those features. In order to reduce dimensionality and quantify the discriminative power of the different measures, we summarized the topographic information by averaging across electrodes and investigated whether the global indexes were able to discriminate NREM sleep from wakefulness (two level analysis) and also specific sleep stages (multilevel analysis). For the two level analysis a Mann-Whitney test explored whether the different markers in the two wakefulness stage (average of ACTIVE W and REST W) significantly differed from NREM sleep (average of N2 and N3). To test whether the measures could differentiate the specific sleep or wakefulness stages, a Friedman test was conducted, with post-hoc analysis using Tukey's honestly significant difference (HSD) test.

In figure 2 changes in normalized power can be seen that are consistent with those commonly reported in the literature: low frequency ( $\delta$ and $\theta$ ) power (rows 1 and 2 of figure 2 ) is dominant in NREM sleep and gradually decreases from SLEEP N3 to ACTIVE W. The two wakefulness states are characterized by a dominant occipital $\alpha$ rhythm (row 3 of figure 2). The power spectrum in all three bands significantly distinguished wakefulness from NREM sleep, as found when averaging the result of $\mathrm{N} 2$ and N3, and comparing these with the average of both stages of wakefulness (rest and active) (p $<10^{-4}$, Mann-Whitney test). However, the normalized $\delta$ and $\theta$ power more efficiently discriminate NREM stages N2 and N3 from sleep N1 and wakefulness. The two-level analysis (sleep N2 and N3 vs. awake) follows the approach used by Massimini et al. (2010). Theoretical models of consciousness and experimental results obtained in sleep, anaesthesia and disorders of consciousness predict that the 
long-range information sharing is essential to maintain consciousness. In agreement with these findings we observed that the average strength of long-range connections in the $\alpha$ band gradually increases in the progression from deep sleep (N3) to ACTIVE W (rows 4 and 5 of figure 2). The difference between sleep and wakefulness is more marked if only a small percentage $(10 \%)$ of strongest connections is included in the analysis (row 5 of figure 2). This index is able to significantly discriminate SLEEP N3 from SLEEP N1 and the two wakefulness stages $(\mathrm{p}<0.01)$, and SLEEP N2 from active wakefulness $(\mathrm{p}<0.01)$.

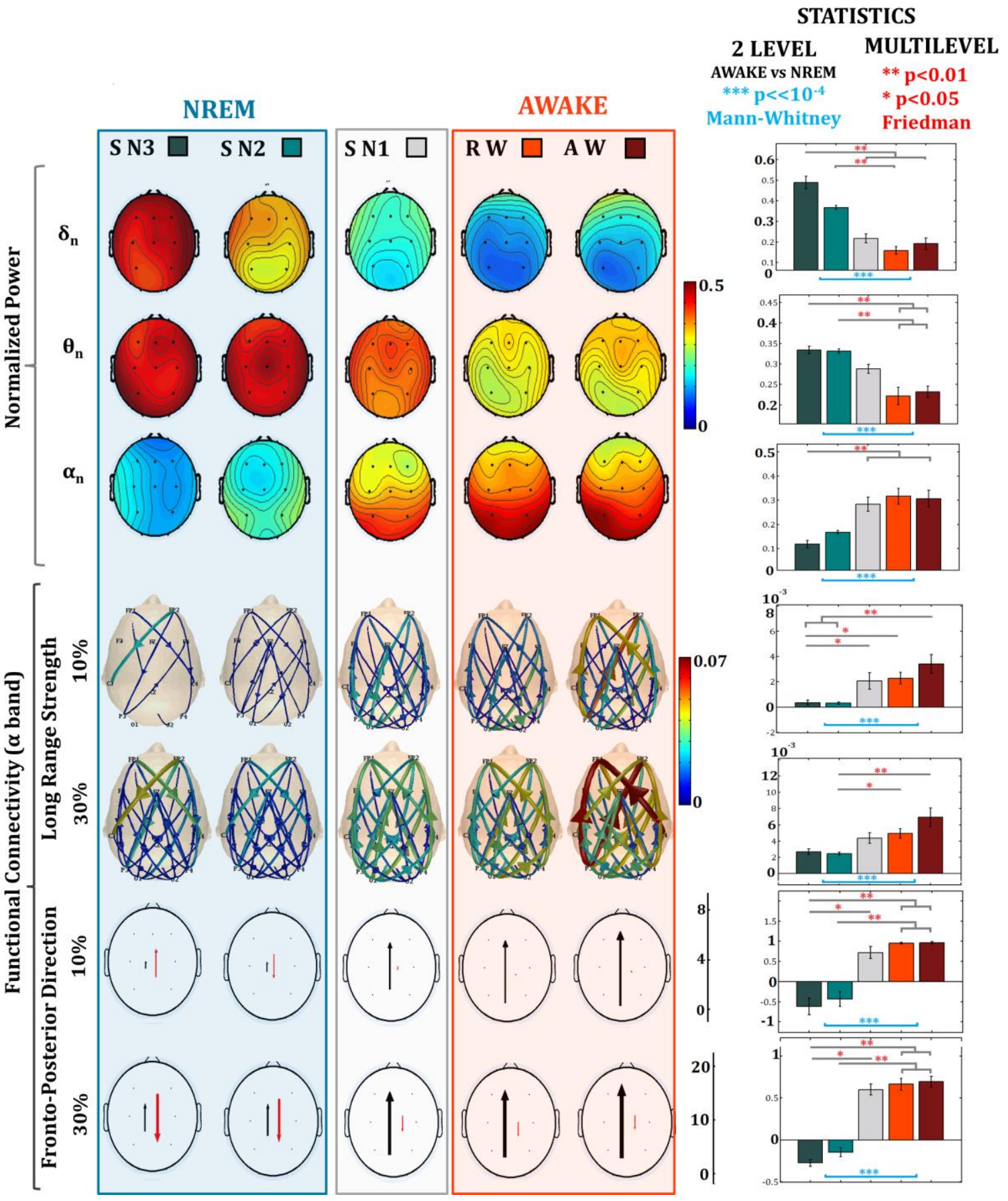

Figure 2. Scalp topography of the different EEG measures (rows), averaged across all 10 subjects, with associated statistics. In the left hand box, rows 1 to 3 show the normalized power distributions $\left(\delta_{n}, \theta_{n}\right.$ and $\left.\alpha_{n}\right)$ across sleep stages. Rows 4 and 5 show long range connections with the Grand Average of the strength of long-range connection in the $\alpha$ band plotted for the $10 \%$ and $30 \%$ strongest connections respectively. Rows 6 and 7 indicate the average number of postero-anterior (black) and antero-posterior (red) connections in the $\alpha$ band coded by the length and thickness of the arrows in the bottom rows for $10 \%$ and $30 \%$ strongest connections respectively. Columns 1 to 5 indicate the 
experimental stage (from SN3 to AW respectively). On the right hand side, the last column indicates whether the indexes averaged across electrodes significantly discriminate wakefulness (REST W and ACTIVE W) from NREM sleep (N2 and N3) as assessed with a two level Mann-Whitney test (the p value is indicated in light blue) and shows results of a multifactor Friedman test on indexes averages across electrodes to assess significant differences across all the stages. The asterisks specify that the two means designated by the brackets significantly differ $(*, \mathbf{p}<0.05 ; * *$, $\mathrm{p}<0.01, * * * \mathrm{p}<10^{-4}$ ), as revealed by Tukey's HSD test (results showed for $10 \%$ strongest connections - fourth row, last column- are repeated from figure 1 , to aid comparison) The connectivity scalp plots were obtained using the eConnectome imaging software (Bin, Astolfi and Babiloni, 2011). Abbreviations: AW-ACTIVE W, RW- REST W, SN1, SN2 and SN3 -NREM sleep stages.

We also assessed changes in the direction of the information flow over the rostro-caudal axis. The most notable trends were observed in the $\alpha$ band (which also showed that greatest changes in connectivity strength as seen in figure 1$)$ and plotted in the two bottom rows ( 6 and 7$)$ of figure 2 . The number of posterior to anterior links dramatically increases from NREM sleep to wakefulness and reaches its maximum in ACTIVE W. In contrast, the number of connection in the antero-posterior direction gradually shrinks in the progression from NREM to wakefulness. Thus the $\operatorname{Dir}_{\mathrm{P}->\mathrm{A}}$ index, that summarizes the dominant direction of information flow, is particularly efficient in discriminating between stages, as revealed by the multilevel analysis. This provides evidence of a significant inversion of information flow in the $\alpha$ band from frontal to posterior vs posterior to frontal in the progression from sleep to wakefulness.
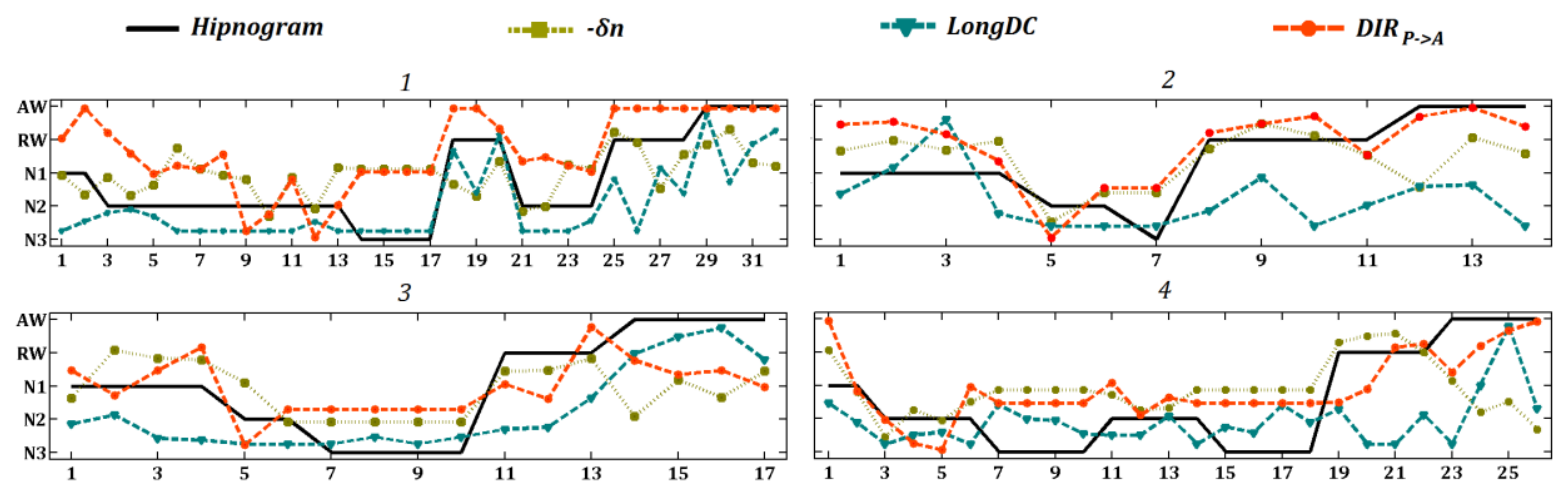

5
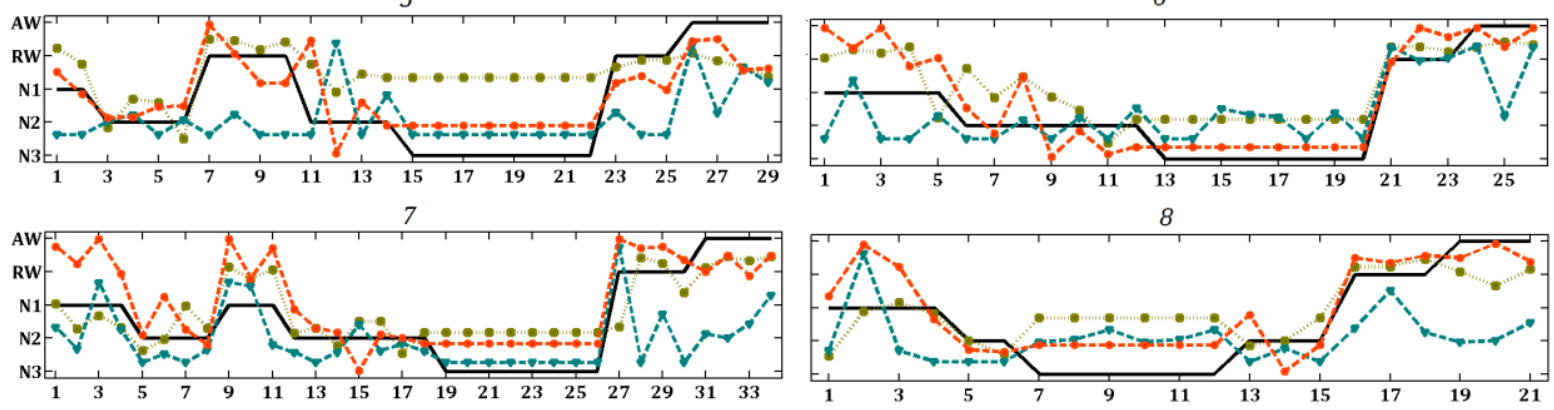

8
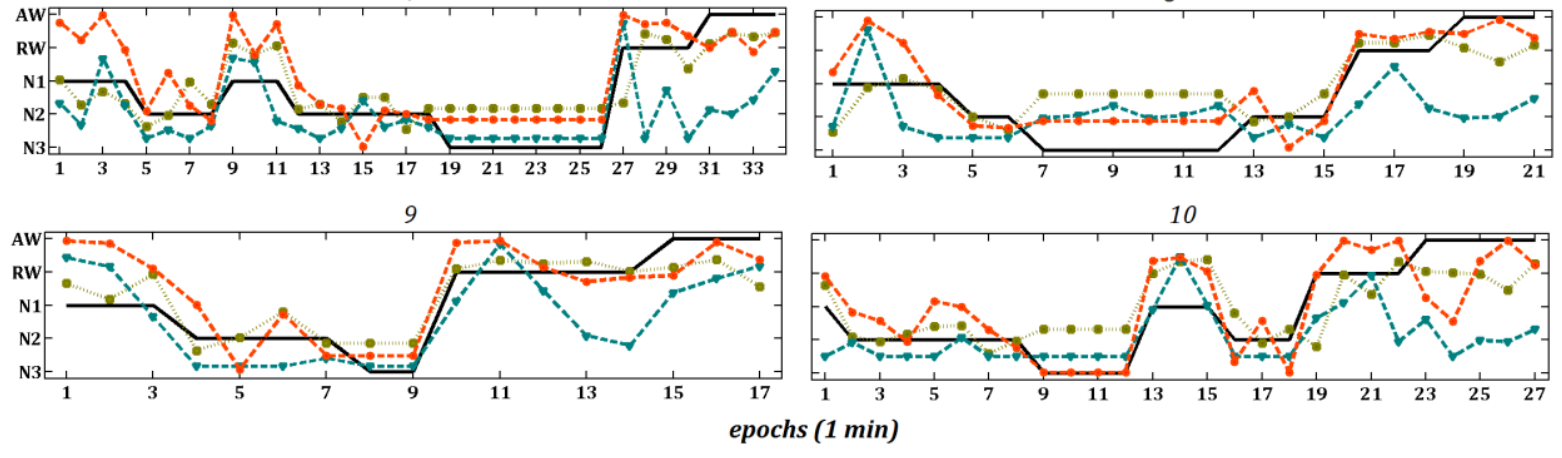

Figure 3. Individual trends over the experimental timeline. Each epoch is 60 seconds in duration. For each of the 10 subjects the amplitude (magnitude squared) of significant long-range DC links (blue dashed line, triangle marker), the Dir ${ }_{P->A}$ index (orange dashed line, circle marker) in the $\alpha$ band and the power in the $\delta$ band (green dotted line, square marker) are plotted and can be compared to the manually scored hypnogram (solid black line). Note that plots are rescaled / inverted to facilitate comparison with the hypnogram. Abbreviations: AW-ACTIVE W, RWREST W, N1, N2 and N3 -NREM sleep stages. For ease of visualization, all plots were rescaled, and delta power was inverted. 


\subsection{Individual analysis}

A clinically useful index of consciousness needs to distinguish between sleep stages at an individual and not only at the group level. In order to investigate whether the changes observed were both consistent at the individual level and able to correlate with the experimental stage across the individual sleep-wake cycle, we show (figure 3) the different indexes for each subject and epoch of the experimental time-line. This epoch by epoch analysis allowed comparison with the individual hypnograms.

Among the connectivity measures, we have plotted the indexes that in the group analysis showed best discriminatory performances: the average strength of the $10 \%$ strongest long-range links and the $\operatorname{Dir}_{P_{\rightarrow A}}$ computed from the $30 \%$ strongest connectivity links. Given that the shift in EEG power toward lower frequencies is a well-known and prominent feature of NREM sleep (sleep $\delta$ waves) and that the $\delta$ and $\theta$ power showed similar discriminative properties at group level, we have only plotted the normalized $\delta$ power from the spectral measures (the plots were inverted to facilitate the comparison with the hypnogram and the connectivity derived indexes). The experimental stages were assigned a value as a measure of LOC, ranging from 0 (SLEEP N3) to 4 (ACTIVE W), and Spearman correlation was computed between each parameter and the individual hypnogram. Table 1 shows the resulting correlation values for individual subjects.

Table 1. Spearman correlation coefficient computed between the hypnogram and each EEG index for individual subjects. Values in bold correspond to a significance level of $p<0.01$, values in bold italic to a significance level $p<0.05$; other values are not significant. Results relative to the $\operatorname{Dir}_{P_{\rightarrow} A}$, long-range $D C$ links and $\delta$ power were highlighted in grey, to facilitate comparison with figure 3. The last column shows the percentage of subjects where the specific EEG index was able to significantly discriminate wakefulness (rest and active) from NREM sleep (N2 and N3) at individual level, as assessed by a Wilcoxon signed rank test $(\mathbf{p}<0.05)$.

\begin{tabular}{|c|c|c|c|c|c|c|c|c|c|c|c|c|}
\hline & \multicolumn{10}{|c|}{ Subject } & \multirow[t]{2}{*}{ Mean \pm Std } & \multirow{2}{*}{$\begin{array}{c}\text { \% subjs } \\
\text { p<0.05 } \\
2 \text { level }\end{array}$} \\
\hline & 01 & 02 & 03 & 04 & 05 & 06 & 07 & 08 & 09 & 10 & & \\
\hline $\begin{array}{c}\text { Norm } \\
\text { Power } \delta\end{array}$ & -0.36 & -0.44 & -0.52 & 0 & -0.67 & -0.89 & -0.79 & -0.64 & -0.83 & -0.63 & $-0.58 \pm 0.26$ & $100 \%$ \\
\hline $\begin{array}{c}\text { Norm } \\
\text { Power } \theta\end{array}$ & -0.54 & -0.15 & -0.51 & -0.38 & -0.50 & -0.72 & -0.76 & -0.87 & -0.55 & -0.80 & $-0.58 \pm 0.2$ & $90 \%$ \\
\hline $\begin{array}{l}\text { Norm } \\
\text { Power } \alpha\end{array}$ & 0.34 & 0.2 & 0.13 & 0.02 & 0.1 & 0.41 & 0.45 & 0.22 & 0.58 & 0.51 & $0.29 \pm 0.18$ & $80 \%$ \\
\hline $\begin{array}{c}\text { Long DC } \\
\alpha(10 \%)\end{array}$ & 0.77 & 0.27 & 0.84 & 0.08 & 0.49 & 0.48 & 0.69 & 0.16 & 0.72 & 0.63 & $0.51 \pm 0.25$ & $70 \%$ \\
\hline $\begin{array}{c}\text { Long DC } \\
\alpha(30 \%)\end{array}$ & 0.83 & 0.21 & 0.75 & 0.51 & -0.01 & 0.63 & 0.38 & -0.23 & 0.75 & 0.46 & $0.43 \pm 0.34$ & $70 \%$ \\
\hline $\begin{array}{l}\operatorname{Dir}_{P_{\rightarrow} A} \\
(10 \%)\end{array}$ & 0.56 & 0.73 & -0.34 & 0.87 & 0.86 & 0.67 & 0.78 & 0.77 & 0.85 & 0.89 & $0.66 \pm 0.37$ & $90 \%$ \\
\hline $\begin{array}{l}\operatorname{Dir}_{P \rightarrow A} \\
(30 \%)\end{array}$ & 0.82 & 0.74 & 0.68 & 0.70 & 0.85 & 0.84 & 0.82 & 0.79 & 0.68 & 0.80 & $0.77 \pm 0.06$ & $100 \%$ \\
\hline
\end{tabular}

All the indexes considered exhibit dramatic changes as a function of the sleep stages at the individual level. As shown in figure 3, the normalized $\delta$ power follows the experimental time-line in the majority of subjects. However in two subjects ( 2 and 4$)$ the changes in $\delta$ power do not track the hypnogram and do not significantly correlate with the LOC (table 1). Similar results are obtained for the $\theta$ power, with a significant negative correlation with the experimental time-line only in 7 of the 10 subjects. The average strength of long-range connections is severely reduced in NREM sleep at an individual level. It shows performances similar to the normalized power indexes, highly correlating with the LOC in 
the majority of subjects, but failing to do so in three of them. Of all the parameters considered, the $\operatorname{Dir}_{P->A}$ showed the best performances in 'tracking' the individual hypnogram, with a high and significant correlation in each of the subjects and the highest mean correlation value. It also is able to significantly discriminate between NREM sleep and wakefulness at an individual level in all the subjects (table 1, last column), as assessed by a Wilcoxon test across the epochs of each stage.

\section{Discussion}

The objective of the current work was to propose and test indexes of brain connectivity that could distinguish between states of consciousness. These indexes should also be computationally relatively simple for clinical application such as in sleep studies or depth of anaesthesia monitoring. Given the conceptual model that the loss of consciousness is associated with a loss in the brain's ability to integrate information (Tononi, 2008), we focussed on connectivity measures, that include directional information. In assessing the performance of these measures, we compared them to the more established power spectral indexes taken from the EEG.

To this end we collected polysomnographic recordings from a sample of 10 healthy subject undergoing post-prandial sleep and extracted the EEG indexes across the sleep-wake cycle at individual and group level. Those indexes were chosen exploiting previous theoretical and experimental findings on neural correlates of consciousness and included normalized power and connectivity based indexes. We found that the proposed amplitude of long-range connections across the scalp and especially the $\operatorname{Dir}_{P_{\rightarrow} A}$ index (that quantifies the dominant direction of information flow in the rostro-caudal axis) showed a monotonic change with the LOC. In the current sample, the $\operatorname{Dir}_{\mathrm{P}_{\rightarrow} \mathrm{A}}$ index showed the best performances in tracking the individual experimental time line, and consistently correlated well with the hypnogram, and significantly discriminated NREM sleep from wakefulness in each of the subjects in the sample. Its performance was found to be superior (MannWhitney test, $\mathrm{p}=0.041$ ) to that of the power in the $\delta$ frequency band, which has been widely used in the past.

\subsection{Methodological considerations}

In this study an advanced method (Directed Coherence) for the estimation of functional connectivity that is able to infer directed causal information was chosen. The direction of connectivity networks has not previously been well explored in finding indexes of LOC, even though it has been suggested that it could provide important insights into neural correlates of consciousness (Sitt et al., 2014). Functional connectivity estimators based on an MVAR model have been shown to be robust to noise (Blinowska 2011) and to perform well even in the case of some non-linear interactions (Winterhalder et al. 2005), and have also been widely applied for estimating functional connectivity from multichannel EEG in different experimental conditions other than sleep (Astolfi et al. 2008; Blinowska 2011). In this context the term 'causality' has been used to refer to Granger causality, i.e. indicating that one signal predicts another in the MVAR model of simultaneously observed signals. We disregarded more complex measures of functional connectivity such as those based on information theory (e.g. transfer entropy) in order to achieve the necessary computational simplicity and temporal resolution required for on-line monitoring (Barnett, Barrett and Seth, 2009).

Despite DC having become well established, caution is required in the interpretation of its results as necessarily indicating causal links connecting underlying cortical sources. Volume conduction effects lead to spreading of electrical activity to a number of electrodes, which could be confused with functional (neurological) connectivity between these brain regions. One distinguishing feature of volume conduction effects is that it is virtually instantaneous, without the delay typical of neuronal 
activity. The supposition that DC is not affected by volume conduction because it is sensitive only to phase differences between channels (Kaminski and Blinowska 2014), is still debated (Faes and Nollo 2011). Our own studies (Lioi et al., in preparation), as well as those of Faes et al.(Faes and Nollo, 2010, 2011; Faes et al., 2013), have suggested that while DC and DTF do not eliminate volume conduction effects, they do reduce them, when compared to conventional coherence. That being said, DC networks estimated during motor (Ginter et al. 2001), attention and memory tasks (Kuś et al. 2008; Brzezicka et al. 2011) show a remarkable agreement with evidence obtained from anatomical consideration and other neuroimaging techniques (Kaminski and Blinowska 2014). In this study, volume conduction effects are less of an issue since the analysis was focused on long-range connectivity, whereas short-range connections (which are likely to be more strongly dominated by the spread due to electrical conduction in tissue and bone) were disregarded. Non-significant connectivity was also removed from the study using surrogate data analysis. Caution must also be exercised in interpreting DC as reflecting direct connections between cortical regions because the method cannot remove the confounding effect of sources (typically deep in the brain) that spread activity to pairs of electrodes on the scalp. From the signals recorded on the scalp it may be impossible to determine whether there are direct neuronal pathways between the corresponding two cortical regions or if both are driven by another (unmeasured) source.

In this exploratory study of connectivity measures, it was important to test the statistical significance of each DC connectivity estimate, which was achieved with surrogate data analysis. This precaution has not always been taken in previous work. However the results showed that the $30 \%$ strongest DC connections calculated from one-minute segments were almost always statistically significant (i.e. DC was larger than obtained with the surrogate data under the null hypothesis of no connections). This suggests that in future work the computationally costly surrogate data analysis may not be required, when using the proposed indexes. This would make real-time implementation computationally feasible. The surrogate data generation takes the $95 \%$ of the total computation time in estimating connectivity. To give an indication of computational cost, if we exclude the shuffling procedure, the time required to estimate connectivity for a $1 \mathrm{~min}$ recording with 12 electrodes and 1 min epochs, is roughly $20 \mathrm{~s}$, when using Matlab® and a typical Windows-based PC.

In the analysis of brain connectivity, the many relatively weak links can obscure important connections. Thresholds are thus usually recommended and applied (Rubinov and Sporns, 2010) to only select connections deemed to be important. The choice of the threshold is somewhat arbitrary (Sporns, 2013), but statistical significance (i.e. a threshold set at the critical value) should always be satisfied. In the current work either the $10 \%$ or the $30 \%$ strongest connections were analysed, following some preliminary investigations. The choice of threshold reflects a compromise between including too many connections that may only be weakly related to the LOC and discarding connections that might hold useful information. Further work in optimizing the threshold for specific tasks such as assessing depth of anaesthesia or sleep stage should be carried out on a larger sample of recordings.

\subsection{The relationship of our findings to previous studies}

To the best of our knowledge there is only one previous study investigating EEG networks using directional measures of connectivity, but this used the closely related approach of DTF (see Methods section) in sleep (Kamiński, Blinowska, and Szclenberger, 1997). DC, which includes information on signal power flowing between different regions of the brain, as well as the transfer function of the linear 'filters' linking these regions (as used in DTF), would seem to be more appropriate than DTF for quantifying the functional connections between brain regions (Baccalà and Sameshima, 2007; Faes and Nollo, 2011). The current paper thus goes beyond previous work in describing DC patterns during the change from wakefulness to NREM sleep, extending the analysis to different frequency 
bands and refining the methods with rigorous statistical significance assessment of the estimated links. Furthermore, we differentiate connectivity links with respect to their inter-electrode distance and we provide an assessment of performance in individual subjects, as well as that at the cohort-level. Our findings are in line with results from the study of Kaminski and colleagues (1997) that showed more complex and denser connections in wakefulness than in NREM sleep and a prevalence of posterior sources during wakefulness. Despite the strong topological similarities, in the study of Kaminski, connectivity networks exhibited a notably larger number of connections, possibly as a result of not performing a significance test (or using a different choice of threshold criterion) for including the estimated DTF in the final analysis, and also because connectivity was integrated over a larger frequency range $(0-30 \mathrm{~Hz})$ than in the current study.

The underlying conjecture of our as well as a number of other studies (Kamiński, Blinowska and Szclenberger, 1997; John and Prichep, 2005; Mashour, 2006; Tononi, 2008) is that changes in LOC are critically associated with a dynamic reorganisation of large-scale connectivity patterns. In line with previous results in disorders of consciousness (Chennu et al., 2014) the connectivity networks in the $\alpha$ band showed the best discriminative performance between sleep stages. Long-range connectivity in the $\alpha$ band was shown to be impaired in NREM sleep, when networks are active but characterized mainly by short-range links. Our results thus support previous studies using different methods and protocols suggesting that the disruption of wakefulness in NREM sleep may be a result of impaired information sharing among cortical areas. Massimini and colleagues (Massimini et al. 2010; Massimini et al. 2005) used a pertubational approach involving Transcranical Magnetic Stimulation (TMS) and EEG recording to investigate how TMS triggered neural activity spread from the stimulation site. During wakefulness the TMS elicited complex patterns of scalp waves spreading to distant cortical areas. During NREM sleep, TMS evoked a stereotypical and local response, thus indicating a 'breakdown of long-range effective connectivity'. The findings of Massimini et al. (2010) and our data suggesting that long range connections reduce during sleep also fit well into a wide range of evidence from a growing literature investigating fMRI brain connectivity in altered states of consciousness such as NREM sleep (Spoormaker et al., 2010; Spoormaker, Gleiser and Czisch, 2012), general anaesthesia (Boly et al. 2011; Schrouff et al. 2011) and vegetative states (Boly and Seth, 2012; King et al., 2013). It appears that those states share, among other major features, a suppression of functional connectivity.

In SLEEP N1 all the EEG indexes showed values intermediate between deeper sleep (N2 and N3) and wakefulness. Often spectral and networks features elicited in SLEEP N1 were more similar to wakefulness than to NREM sleep. SLEEP N1 represents the transition between wakefulness and sleep and it is considered "unstable sleep" (Klimova, 2014). When awakened from SLEEP N1 subjects often report dream-like experiences or claim they were awake (Nir, Massimini and Boly, 2013). Experimental results suggest preserved long-range connectivity in this stage (Massimini et al., 2005). For this reason we have considered only sleep N2 and N3 trials to characterize stable NREM sleep in the two level analysis, as used in Massimini et al. (2010).

The findings of recent seminal works in Network Physiology (Bashan et al., 2012; Bartsch et al., 2015; Liu et al., 2015) broadly align with our study. Network physiology is a new research field that aims to characterize how the various physiological systems dynamically integrate their functions in different physiological (and pathological) states (Bashan et al., 2012; Ivanov, Liu and Bartsch, 2016). A series of studies investigating changes in networks of interactions between (Bartsch et al., 2015) and within (Liu et al., 2015) different physiological systems (muscular, cardiac, respiratory and central nervous systems) during the transition from wakefulness to sleep and across sleep stages have provided important progress in this emergent field, contributing to the realization of an atlas of global network physiology in sleep and wakefulness. In these studies network connectivity was assessed using an estimator based on the stability of the time delay between signals (time delay stability -TDS), which quantifies in fine temporal detail the undirected strength of coupling. Despite the differences in 
approach, EEG networks estimated from six channels and in different frequency bands in Liu et al. 2015 and Bartsch et al. 2015, showed important similarities with those shown in our study for the $\alpha$ band: they observed a significant decrease in the strength and number of links (in particular of longrange fronto-occipital connections) in deep sleep (as compared to wakefulness and light sleep) and a remarkable symmetry between the two hemispheres, characterizing all physiological states. Another notable analogy with our results regards EEG networks elicited in sleep N1, whose features are more similar to wakefulness than to deeper sleep. As Liu and colleagues have observed, this is an interesting result, given that sleep N1 is commonly classified as belonging to the same macro state (NREM sleep) as sleep N2 and N3. In their analysis of brain networks Liu and colleagues have also reported that while local connections (frontal/frontal, central/central and occipital/occipital) are reduced but preserved in deep sleep, fronto-occipital and occipital-frontal networks show practically no connection in deep sleep: these findings agree clearly with the significant impairment of longrange connectivity we observed in sleep N2 and N3.

Our study provides important new contributions beyond this work (and previous studies) in the characterization of brain networks by assessing the direction of links and the consequent ability to identify a switch in the direction of information flow with sleep onset, that constitutes the most characteristic change in DC patterns. Group analysis reveals a significant inversion of the direction of posterio-frontal networks with state. The marked posterior to anterior spread of $\alpha$ rhythm in wakefulness is reversed in NREM sleep (N2 and N3) that is characterized by a dominance of frontal sources of activity. An inversion of information flow from frontal-posterior in sleep to posteriorfrontal in wakefulness has also been found in a previous analysis of sleep and general anaesthesia onset (De Gennaro et al., 2004; Nicolaou et al., 2012).

The importance of quantifying, together with the strength, the direction of links has been highlighted in a recent work where the TDS estimator originally proposed in the framework of network physiology has been extended to the concept of delay-correlation landscape (DCL) (Lin et al., 2016). While the TDS is computed considering the delay corresponding to the maximum (absolute) correlation between signals, the DCL estimator retains information about the delay dependence of the cross-correlation, which carries important information about the directionality of physiologic interactions. The DCL approach has been specifically designed to quantify interactions between the outputs of different physiological systems and it was shown to efficiently grasp the directed correlation between EEG power in different bands and the cardiac signal (Lin et al., 2016). If, however, the aim of the analysis is to investigate interactions between signals of the same subsystem (brain-brain in this case), DC may be more efficient in capturing the direction and strength of links as it summarizes the different delays dependences using a simple metric (direction of links) that can be synthetically represented with a directed arrow. On the other hand, in the case of DCL the directional information is coded in a more complex and rich 'landscape' of different delays for positive and negative correlations. While conveying important information about the type of dependence (negative or positive) at different time lags, the DCL estimator is probably more difficult to interpret and present in a succinct manner. In addition, in the case of EEG signals, correlation (including DCL, as well as the coherence in the frequency domain) is likely to be more heavily affected by confounding volume conduction effects than the DC (Faes et al., 2013).

In view of an application in the framework of Network Physiology, DC presents important features that are supplementary to the proposed measures based on time-delays. In the case of EEG signals, the two estimators measure different aspects of the interaction between signals. While TDS quantifies connectivity (as stable time delay) between EEG spectral power (brain rhythms) in different frequency bands, the DC quantifies linear connectivity (Granger causality) between channels of the original EEG time series and then transform it into the frequency domain. The application of DC may be therefore promising for the analysis of sub-system connectivity and provide new insights related to the network physiology atlas in physiological and pathological states. DC is based on linear modelling, 
and may thus outperform alternatives when this assumption is (approximately) valid, but is likely to underperform when the assumption is grossly violated. Further work is required to test the application of the MVAR approach to model interaction between signals on different time scales and from different physiological systems.

Another important original contribution of this work is the investigation, and presentation, of patterns for individual subjects, in addition to aggregated cohort results. The majority of studies investigating EEG markers of LOC have only performed group analysis or found the disagreement between behavioural and EEG-based measures too high for a reliable individual assessment (Sitt et al., 2014). Our long-term aim is to identify an EEG index able to 'track' the consciousness level of the subjects, with a view to assess depth of sleep or anaesthesia. The performance of the proposed measures in individuals provides an indication of the potential of the proposed approach.

Among all the markers considered in the current paper, the $\operatorname{Dir}_{\mathrm{P}_{\rightarrow} \mathrm{A}}$ index was found to be the most reliable in tracking the LOC during individual sleep experiments, strongly correlating with the hypnogram in all the subjects. It should be noted that the staging of the EEG time series was performed taking in account the proportion of $\delta$ waves, as recommended in standard sleep staging criteria, therefore high correlation between measured $\delta$ power and sleep stages is only to be expected. Given that EEG spectral features were used to characterize sleep (Corsi-Cabrera et al., 2003) and to monitor hypnotic level in anaesthesia (Myles et al., 2004), it is interesting that the $\operatorname{Dir}_{P_{\rightarrow} \mathrm{A}}$ index, which reflects very different features of the EEG signals, showed better performance than the $\delta$ power in correlating with the sleep stage at an individual level. These results suggest that the inversion of information flow represents a promising indicator of the descent into deep sleep. We speculate that this may also be seen in other states of altered consciousness, such as anaesthesia. In order to arrive at an even more powerful index of LOC, it may be beneficial to combine power-spectral measures with those obtained from DC and this approach is currently being pursued.

\subsection{Limitations}

The current work was carried out on a relative small sample (10 subjects) recorded during a nap, not all-night sleep. Thus only between 13 and 31 one-minute segments were available from each subject in this cohort (see figure 3). While the results showed the power of the $\operatorname{Dir}_{\mathrm{P} \rightarrow \mathrm{A}}$ index, this could be specific to this small sample. The current work should thus be considered as exploratory, in which the hypothesis of superior performance of $\operatorname{Dir}_{\mathrm{P} \rightarrow \mathrm{A}}$ was generated, but cannot be robustly tested, on the same small sample. Further tests on an independent sample, that might include a wider range of indexes as well as their combination should be carried out to independently statistically test relative performance measures.

\section{Conclusions}

We have assessed the performances of different EEG indexes to predict the level of consciousness during a NREM sleep study. Our results show that the EEG signal includes many features able to discriminate NREM sleep from wakefulness at a group level, but correlation with individual hypnograms varied across subjects. In agreement with theoretical consideration, EEG indexes relying on directional connectivity assessment have proven to be particular promising. Among all of the EEG measures tested, a proposed index of the direction of information flow on the rostro-caudal axis that is based on Directed Coherence, performed well at a group level and gave the highest correlation with individuals' level of consciousness. 


\section{Acknowledgements}

This work was supported by the National Institute of Academic Anaesthesia (UK) and the Kerkut Trust (University of Southampton, UK).

\section{References}

Achermann, P. and Borbély, A. (1998) 'Coherence analysis of the human sleep electroencephalogram', Neuroscience, 85(4), pp. 1195-1208.

Baccalá, L. A., Sameshima, K., Ballester, G., Do Valle, a. C. and Timo-Iaria, C. (1998) 'Studying the Interaction Between Brain Structures via Directed Coherence and Granger Causality', Applied Signal Processing, 5(1), pp. 40-48.

Baccalà, L. A. and Sameshima, K. (2007) 'Generalized Partial Directed Coherence', in Proc. of the 2007 15th Intl. Conf. on Digital Signal Processing, pp. 1-4.

Barnett, L., Barrett, A. B. and Seth, A. K. (2009) 'Granger causality and transfer entropy Are equivalent for gaussian variables', Physical Review Letters, 103(23), pp. 1-10.

Bartsch, R. P., Liu, K. K. L., Bashan, A. and Ivanov, P. C. (2015) 'Network physiology: How organ systems dynamically interact', PLoS ONE, 10(11), pp. 1-34.

Bashan, A., Bartsch, R. P., Kantelhardt, J. W., Havlin, S. and Ivanov, P. C. (2012) 'Network physiology reveals relations between network topology and physiological function', Nature Communications. Nature Publishing Group, 3, pp. 702-709.

Benjamini, Y. and Yekutieli, D. (2001) 'The control of the false discovery rate in multiple testing under depencency', The Annals of Statistics, 29(4), pp. 1165-1188.

Bin, H., Astolfi, L. and Babiloni, F. (2011) 'eConnectome: a MATLAB toolbox for mapping and Imaging brain functional connectivity', J Neurosci Methods, 195(2), pp. 261-269.

Boly, M., Garrido, M. I., Gosseries, O., Bruno, M.-A., Boveroux, P., Schnakers, C., Massimini, M., Litvak, V., Laureys, S. and Friston, K. (2011) 'Preserved feedforward but impaired top-down processes in the vegetative state.', Science (New York, N.Y.), 332(6031), pp. 858-62.

Boly, M., Perlbarg, V., Marrelec, G., Schabus, M., Laureys, S., Doyon, J., Pelegrini-Issac, M., Maquet, P. and Benali, H. (2012) 'Hierarchical clustering of brain activity during human nonrapid eye movement sleep', Proceedings of the National Academy of Sciences, 109(15), pp. 5856-5861.

Boly, M. and Seth, A. K. (2012) 'Modes and models in disorders of consciousness science.', Archives italiennes de biologie, 150(2-3), pp. 172-84.

Brooks, A. and Lack, L. (2006) 'A brief afternoon nap following nocturnal sleep restriction: which nap duration is most recuperative?', Sleep, 29(6), pp. 831-840.

Brown, E. N., Lydic, R. and Shiff, N. D. (2010) 'General Anesthesia, Sleep and Coma', The New England Journal of Medicine, 363(27), pp. 2638-50.

Chennu, S., Finoia, P., Kamau, E., Allanson, J., Williams, G. B., Monti, M. M., Noreika, V., Arnatkeviciute, A., Canales-Johnson, A., Olivares, F., Cabezas-Soto, D., Menon, D. K., Pickard, J. D., Owen, A. M. and Bekinschtein, T. A. (2014) 'Spectral Signatures of Reorganised Brain Networks in Disorders of Consciousness', PLoS Computational Biology, 10(10).

Corsi-Cabrera, M., Miró, E., Del-Río-Portilla, Y., Pérez-Garci, E., Villanueva, Y. and Guevara, M. a. (2003) 'Rapid eye movement sleep dreaming is characterized by uncoupled EEG activity between 
Directional connectivity in the EEG is able to discriminate wakefulness from NREM sleep

frontal and perceptual cortical regions', Brain and Cognition, 51(3), pp. 337-345.

Faes, L., Erla, S., Porta, A. and Nollo, G. (2013) 'A framework for assessing frequency domain causality in physiological time series with instantaneous effects', Phil. Trans. R. Soc. A, 371.

Faes, L. and Nollo, G. (2010) 'Extended causal modeling to assess Partial Directed Coherence in multiple time series with significant instantaneous interactions.', Biological cybernetics, 103(5), pp. $387-400$.

Faes, L. and Nollo, G. (2011) 'Multivariate Frequency Domain Analysis of Causal Interactions in Physiological Time Series', Biomedical Engineering, Trends in Electronics, Communications and Software. Edited by Mr Anthony Laskovski (Ed.). ISBN: 978-953-307-475-7, InTech.

Ferrarelli, F., Massimini, M., Sarasso, S., Casali, A., Riedner, B. a, Angelini, G., Tononi, G. and Pearce, R. a (2010) 'Breakdown in cortical effective connectivity during midazolam-induced loss of consciousness.', Proceedings of the National Academy of Sciences of the United States of America, 107(6), pp. 2681-2686.

Ferri, R., Rundo, F., Bruni, O., Terzano, M. G. and Stam, C. J. (2005) 'Dynamics of the EEG slowwave synchronization during sleep', Clinical Neurophysiology, 116(12), pp. 2783-2795.

Ferri, R., Rundo, F., Bruni, O., Terzano, M. G. and Stam, C. J. (2007) 'Small-world network organization of functional connectivity of EEG slow-wave activity during sleep', Clinical Neurophysiology, 118(2), pp. 449-456.

De Gennaro, L., Vecchio, F., Ferrara, M., Curcio, G., Rossini, P. M. and Babiloni, C. (2004) 'Changes in fronto-posterior functional coupling at sleep onset in humans.', Journal of sleep research, 13(3), pp. 209-17.

Gómez, F., Phillips, C., Soddu, A., Boly, M., Boveroux, P., Vanhaudenhuyse, A., Bruno, M.-A., Gosseries, O., Bonhomme, V., Laureys, S. and Noirhomme, Q. (2013) 'Changes in effective connectivity by propofol sedation.', PloS one, 8(8), p. e71370.

De Graaf, T. A., Hsieh, P. J. and Sack, A. T. (2012) 'The "correlates" in neural correlates of consciousness', Neuroscience and Biobehavioral Reviews, 36(1), pp. 191-197.

Hlaváčková-Schindler, K. (2011) 'Equivalence of Granger Causality and Transfer Entropy: A Generalization', Applied Mathematical Sciences, 5(73), pp. 3637-3648.

Iber, C., Ancoli-Israel, S., Chesson, A. L. and Quan, S. F. (2007) The AASM Manual for the Scoring of Sleep and Associated Events. Wesrchester, IL, 60154, U.S.A.

Ivanov, P. C., Liu, K. K. L. and Bartsch, R. P. (2016) 'Focus on the emerging new fields of network physiology and network medicine', New Journal of Physics. IOP Publishing, 18(100201).

John, E. R. and Prichep, L. S. (2005) 'The Anesthetic Cascade. A Theory of How Anesthesia Suppresses Consciousness', Anesthesiology, 102(2), pp. 447-471.

Kaminiski, M. J. and Blinowska, K. J. (1991) 'A new method of the description of the information flow sources’, Biological cybernetics, 65(3), pp. 203-210.

Kaminski, M. and Blinowska, K. J. (2014) 'Directed Transfer Function is not influenced by volume conduction-inexpedient pre-processing should be avoided.', Frontiers in computational neuroscience, 8(June), p. 61.

Kamiński, M., Blinowska, K. and Szclenberger, W. (1997) 'Topographic analysis of coherence and propagation of EEG activity during sleep and wakefulness.', Electroencephalography and clinical neurophysiology, 102(3), pp. 216-27.

King, J.-R., Sitt, J. D., Faugeras, F., Rohaut, B., El Karoui, I., Cohen, L., Naccache, L. and Dehaene, S. (2013) 'Information sharing in the brain indexes consciousness in noncommunicative patients.', 
Directional connectivity in the EEG is able to discriminate wakefulness from NREM sleep

Current biology : CB, 23(19), pp. 1914-9.

Klimesch, W. (1999) 'EEG alpha and theta oscillations reflect cognitive and memory performance: a review and analysis.', Brain research. Brain research reviews, 29(2-3), pp. 169-95.

Klimova, M. (2014) 'What Is Lost During Dreamless Sleep : The Relationship Between Neural Connectivity Patterns and Consciousness', Journal of European Psychology Students, 5(3), pp. 56-65.

Koch, C., Massimini, M., Boly, M. and Tononi, G. (2016) 'Neural correlates of consciousness: progress and problems', Nature Reviews Neuroscience, 17(5), pp. 307-321.

Lin, A., Liu, K. K. L., Bartsch, R. P. and Ivanov, P. C. (2016) 'Delay-correlation landscape reveals characteristic time delays of brain rhythms and heart interactions', Phil. Trans. R. Soc. A, 374(20150182).

Lioi, G., Bell, S. L. and Simpson, D. M. (2016) 'XIV Mediterranean Conference on Medical and Biological Engineering and Computing 2016: MEDICON 2016, March 31st--April 2nd 2016, Paphos, Cyprus', in Kyriacou, E., Christofides, S., and Pattichis, C. S. (eds). Cham: Springer International Publishing, pp. 3-8.

Liu, K. K. L., Bartsch, R. P., Lin, A., Mantegna, R. N. and Ivanov, P. C. (2015) 'Plasticity of brain wave network interactions and evolution across physiologic states', Frontiers in Neural Circuits, 9(62), pp. 1-15. doi: 10.3389/fncir.2015.00062.

Marinazzo, D., Gosseries, O., Boly, M., Ledoux, D., Rosanova, M., Massimini, M., Noirhomme, Q. and Laureys, S. (2014) 'Directed Information Transfer in Scalp Electroencephalographic Recordings: Insights on Disorders of Consciousness', Clinical EEG and Neuroscience, 45(1), pp. 33-39.

Mashour, G. A. (2006) 'Integrating the science of consciousness and anesthesia', Anesthesia and Analgesia, 103(4), pp. 975-982.

Massimini, M., Ferrarelli, F., Huber, R., Esser, S. K., Singh, H. and Tononi, G. (2005) 'Breakdown of cortical effective connectivity during sleep.', Science (New York, N.Y.), 309(5744), pp. 2228-32.

Massimini, M., Ferrarelli, F., Murphy, M. J., Huber, R., Riedner, B. A., Casarotto, S. and Tononi, G. (2010) 'Cortical reactivity and effective connectivity during REM sleep in humans', Cognitive Neuroscience, 1(3), pp. 176-183.

Mhuircheartaigh, N. R., Warnaby, C., Rogers, R., Jbabdi, S. and Tracey, I. (2013) 'Slow-wave activity saturation and thalamocortical isolation during propofol anesthesia in humans.', Science translational medicine, 5(208), p. 208ra148.

Myles, P. S., Leslie, K., McNeil, J., Forbes, a and Chan, M. T. V (2004) 'Bispectral index monitoring to prevent awareness during anaesthesia: the B-Aware randomised controlled trial.', Lancet, 363(9423), pp. 1757-63.

Nicolaou, N., Hourris, S., Alexandrou, P. and Georgiou, J. (2012) 'EEG-based automatic classification of "awake" versus "anesthetized" state in general anesthesia using granger causality', PLOS ONE, 7(3).

Nir, Y., Massimini, M. and Boly, M. (2013) Neuroimaging of Consciousness Springer-Verlag Berlin Heidelberg.

Rechtschaffen, A. and Kales, A. (1968) A manual of standardized terminology, techniques, and scoring system for sleep stages of human subjects. Edited by Washington Public Heath Service. Washington: US Government Printing Office.

Rubinov, M. and Sporns, O. (2010) 'Complex network measures of brain connectivity: Uses and interpretations’, NeuroImage. Elsevier Inc., 52(3), pp. 1059-1069.

Schlogl, A. and Supp, G. (2006) 'Analyzing event -related EEG data with multivariate autoregressive 
parameters', Prog Brain Res., 159, pp. 135-147.

Schrouff, J., Perlbarg, V., Boly, M., Marrelec, G., Boveroux, P., Vanhaudenhuyse, A., Bruno, M.-A., Laureys, S., Phillips, C., Pélégrini-Issac, M., Maquet, P. and Benali, H. (2011) 'Brain functional integration decreases during propofol-induced loss of consciousness', NeuroImage. Elsevier Inc., 57(1), pp. 198-205.

Sergent, C., Baillet, S. and Dehaene, S. (2005) 'Timing of the brain events underlying access to consciousness during the attentional blink', Nature neuroscience, 8(10), pp. 1391-1400.

Siclari, F., LaRocque, J. J., Bernardi, G., Postle, B. R. and Tononi, G. (2014) 'The neural correlates of consciousness in sleep: a no-task, within-state paradigm', bioRxiv, p. 12443.

Sitt, J. D., King, J. R., El Karoui, I., Rohaut, B., Faugeras, F., Gramfort, A., Cohen, L., Sigman, M., Dehaene, S. and Naccache, L. (2014) 'Large scale screening of neural signatures of consciousness in patients in a vegetative or minimally conscious state', Brain, 137(8), pp. 2258-2270.

Spoormaker, V. I., Gleiser, P. M. and Czisch, M. (2012) 'Frontoparietal connectivity and hierarchical structure of the brain's functional network during sleep', Frontiers in Neurology, MAY(May), pp. 110.

Spoormaker, V. I., Schröter, M. S., Gleiser, P. M., Andrade, K. C., Dresler, M., Wehrle, R., Sämann, P. G. and Czisch, M. (2010) 'Development of a Large-Scale Functional Brain Network during Human Non-Rapid Eye Movement Sleep', J. Neurosci., 30(34), pp. 11379-11387.

Sporns, O. (2013) 'Structure and function of complex brain networks', Dialogues Clin Neurosci, 15, pp. 247-262.

Stickgold, R., Malia, A., R, F., Propper, R. and Hobson, J. A. (2001) 'Brain-mind states: I.

Longitudinal field study of sleep/wake factors influencing mentation report length', Sleep, 24(2), pp. 171-179.

Tagliazucchi, E., von Wegner, F., Morzelewski, A., Brodbeck, V., Jahnke, K. and Laufs, H. (2013) 'Breakdown of long-range temporal dependence in default mode and attention networks during deep sleep.', Proceedings of the National Academy of Sciences of the United States of America, 110(31), pp. 15419-24.

Tononi, G. (2008) 'Consciousness as Integrated Information', The Biological Bulletin, 215(December), pp. 216-242.

Tononi, G. and Massimini, M. (2008) 'Why does consciousness fade in early sleep?', Annals of the New York Academy of Sciences, 1129, pp. 330-334.

Toppi, J., Petti, M., De Vico Fallani, F., Vecchiato, G., Maglione, a G., Cincotti, F., Salinari, S., Mattia, D., Babiloni, F. and Astolfi, L. (2012) 'Describing relevant indices from the resting state electrophysiological networks.', CConf Proc IEEE Eng Med Biol Soc., 2012(1), pp. 2547-50. 